\title{
High-dose rate brachytherapy for palliative care in rectal cancer: A case with a complete response, followed by a rare complication
}

\author{
Aurélia Alati ${ }^{*}$, Leobon Sophie ${ }^{2}$ and Clavère Pierre ${ }^{1}$ \\ ${ }^{1}$ Department of Radiation Oncology, University Hospital, Limoges, France \\ ${ }^{2}$ Department of Medical Oncology, University Hospital, Limoges, France
}

\begin{abstract}
Purpose: There are no guidelines for palliative care to relieve patients from pain, bleeding and obstruction due to locally advanced rectal tumours. These patients are fragile, with comorbidities, and have been previously treated by external-beam radiotherapy. Brachytherapy with iridium-192 (interstitial or endoluminal) delivers a high dose of radiation to the tumour, while preserving the surrounding tissues. This technique achieves effective palliation in many cancers; it is also used worldwide for recurrent or inoperable rectal tumours. We report a patient diagnosed with inoperable rectal adenocarcinoma. He had received endorectal high-dose rate brachytherapy to reduce rectal bleeding and pelvic pain. Nevertheless, he presented a rare complication after brachytherapy.
\end{abstract}

Method: We selected a patient who had been treated with endorectal high-dose brachytherapy at the Limoges University Hospital Centre. His medical records were analysed retrospectively. The patient had severe cardiovascular comorbidities and had previously received pelvic external-beam radiotherapy. High-dose rate brachytherapy was delivered with an endoluminal vaginal/rectal cylinder applicator connected to an iridium-192 stepping source. A single dose of 5 Gy, weekly, was applied. The aim was to improve his quality of life by relieving him of bleeding and pain.

Results: High-dose rate brachytherapy was immediately well tolerated. Moreover, there was no problem of compliance because of the short duration of treatment, which was easily performed. The patient described an overall complete response to his symptoms after 1 month. The bleeding stopped and he was withdrawn from opioids. He regained his quality of life and maintained independence at home. Nevertheless, he died 2 months after the end of the treatment of necrotizing fasciitis of the perineum.

Conclusion:Intraluminal high-dose rate brachytherapy is a very effective palliative treatment after external-beam radiotherapy. This technique can be easily performed to reduce bleeding, pain and obstruction with significant improvement in patient quality of life. However, precautions must be taken for patients with severe peripheral arterial disease who have previously received external-beam radiotherapy.

\section{Introduction}

No standard of palliative care is available for patients with advanced rectal cancer who cannot undergo radical therapy. High-dose rate brachytherapy (interstitial or intraluminal) with Iridium-192 has demonstrated efficiency in palliative treatment of rectal cancer to relieve patients from pain, obstruction and bleeding. However limited data are available, on a small number of patients. We report a case of a patient treated by intraluminal high-dose rate brachytherapy for advanced rectal adenocarcinoma. The aim was to improve his quality of life by relieving rectal bleeding and pelvic pain. He presented with necrotizing fasciitis of the perineum, a rare complication that may involve radiation therapy.

The case was an 84-year-old patient with stage IV obliterative arteriopathy of the lower limbs (aortobifemoral prosthesis) and ischemic cardiopathy (quadruple bypass), who was taking an antiplatelet drug. A rectal tumour was diagnosed in the context of rectal bleeding in February 2006. An investigation discovered a middle-low rectal Lieberkuhnian hemicircumferential adenocarcinoma, extending $50 \mathrm{~cm}$ from the anal margin with locoregional extension to inguinal lymph nodes and mesorectum (Figure 1). He did not present with an altered general condition and was performance status 1 . His major symptom was daily rectal bleeding causing chronic symptomatic anaemia. He had received several argon plasma coagulation treatments between February 2006 and April 2007. Palliative external-beam radiotherapy was based on the persistence of symptomatic anaemia due to bleeding. Radiotherapy was delivered on the rectal volume, and the prescribed dose was $60 \mathrm{~Gy}$ in 30 fractions over 47 days between July and September 2007. The 1-month clinical examination revealed a twothirds reduction in the tumour and a complete abolition of rectorragy. He did not complain of pelvic pain and walking was undisturbed. His main handicap was related to arteriopathy. In February 2008, the patient started to complain of severe perineal pain, justifying the prescription of strong opioids: $10 \mathrm{mg}$ morphine and $100 \mu \mathrm{g}$ /hour fentanyl patch with nefopam. An abdominopelvic scan showed that the rectal tumour was stable (Figure 2). In June 2008, his general condition had worsened to

${ }^{*}$ Correspondence to: Aurélia Alati, Department of Radiation Oncology, University Hospital, Limoges, France, Tel: +33 555056269; Fax: +33555056319; E-mail: aurelia.alati@chu-limoges.fr

Key words: palliative care, external-beam radiotherapy, brachytherapy, iridium-192

Received: March 22, 2019; Accepted: April 12, 2019; Published: April 16, 2019 

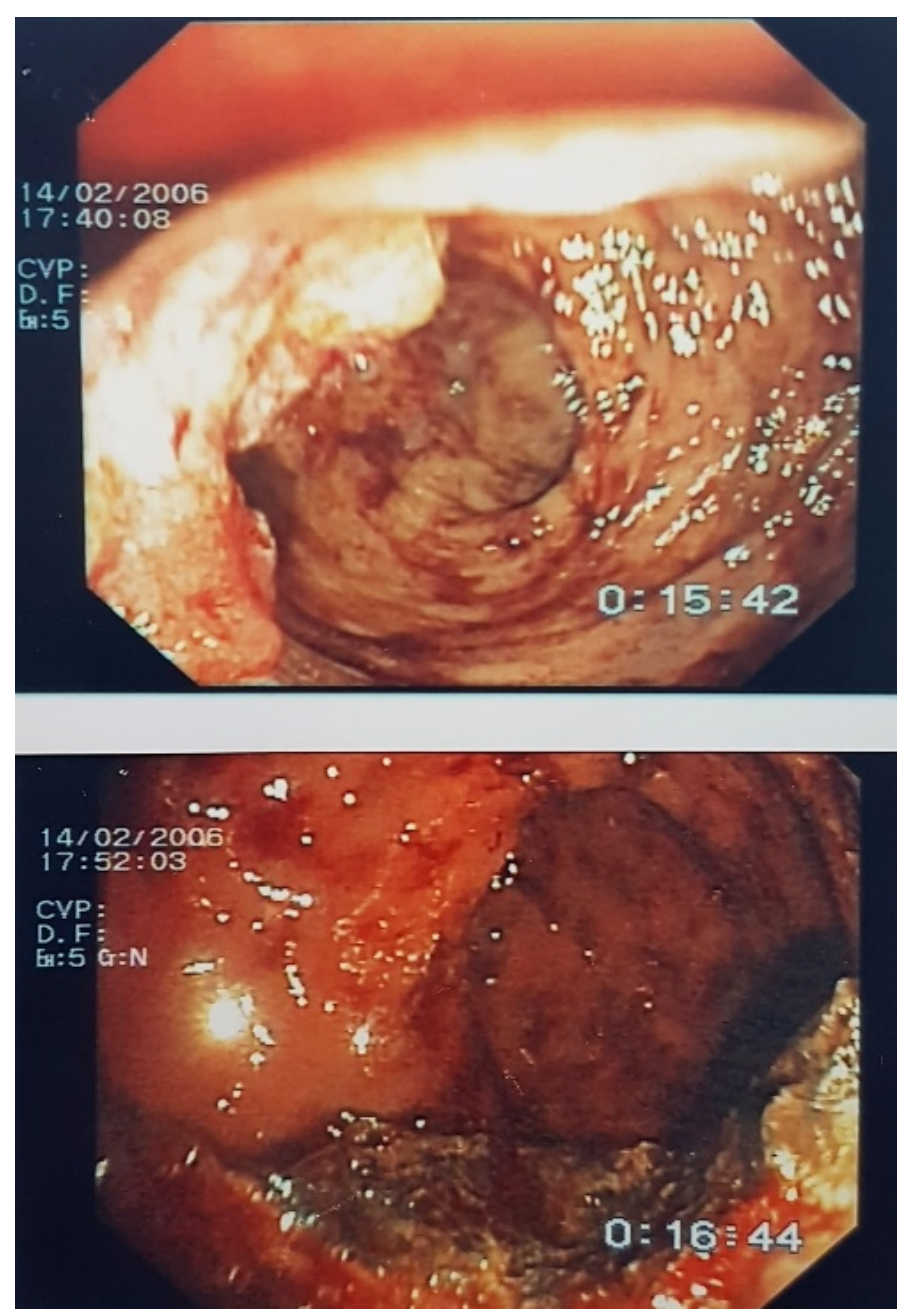

Figure 1. Rectosigmoidoscopy. April 2006 Rectal adenocarcinoma at diagnosis.
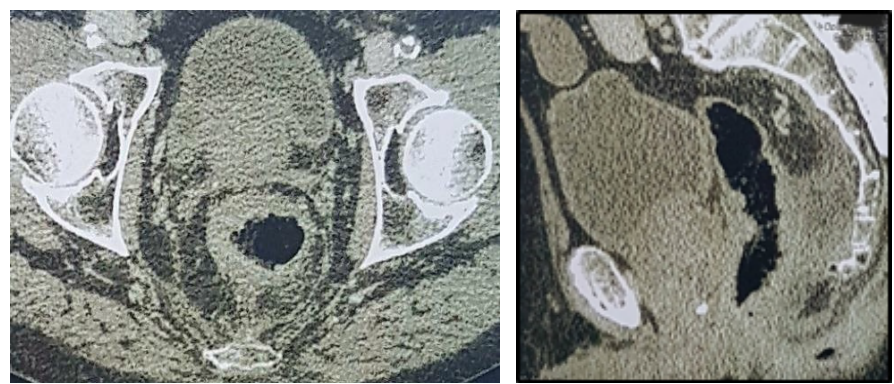

Figure 2. Abdominopelvic scan.

performance status 3. He described urinary and anal incontinence, and strong opioids were insufficient. In addition, rectal bleeding had begun, with the tumour exteriorised in the anal canal.

He suffered from chronic anaemia that required regular blood transfusions. Endoluminal high-dose rate brachytherapy was decided for treatment. Under local anaesthesia, the treatment was composed of a single dose of $5 \mathrm{~Gy}$ per week. He received four focal applications between August and September 2008. He did not present with any adverse effects during the early follow-up period. Nevertheless, he died 2 months later of necrotizing fasciitis of the perineum in our palliative unit. The diagnosis was defined by a physical examination and the patient's risk factors. He presented with rapidly progressing and painful erythema of the perineum in a septic context. Necrotic lesions and cutaneous crepitus on palpation had appeared. Palliative care was decided with palliative sedation.

\section{Patients and methods}

Three patients were treated with rectal endoluminal high-dose rate brachytherapy at the Limoges University Hospital Centre between 2003 and 2018. We selected one patient who had a complete response after brachytherapy but also developed a rare secondary toxicity, such as necrotizing fasciitis of the perineum, which may have involved the radiation therapy. We analysed his medical records retrospectively. $\mathrm{He}$ had an advanced hemicircumferential rectal tumour ( $\geq$ stage III, TNM 8th edition). The diagnosis and staging were determined by a clinical examination, endoscopy, rectal-IRM, abdominopelvic scan and a blood test. First, he received external-beam- radiotherapy followed by high-dose rate brachytherapy for palliative purposes. Pelvic conformal external-beam radiotherapy was delivered with a Clinac 2300 linear accelerator at $25 \mathrm{Mv}$. We used an anterior, a posterior and two lateral beams (Figure 3 and 4). Referring to the second edition of the Recorad guidelines (10), with exclusive radiotherapy, the equivalent dose can reach 55-65 Gy, limited by the rectal volume.

The PTV of 60 Gy covered the rectal volume only, and 2 Gy was delivered daily 5 days per week. The biologically effective dose was $(\alpha / \beta$ rectum: 5) 84 Gy. The dose given in the PTV was superior to $95 \%$ of the prescribed dose and inferior to $107 \%$.

Organ-at-risk dose-constraints were respected (dose-volume histogram). D2\% of the rectal volume did not exceed the prescribed dose.

Scan-based planning was used for dosimetry for the high-dose rate brachytherapy. The patient received four single application doses of 5 Gy per week, using a vaginal/rectal cylinder applicator, diameter $=20$ $\mathrm{mm}$, length $=9 \mathrm{~cm}$ (Figure 3) connected to an iridium-192 stepping source. The procedure did not require a general anaesthetic. The PTV prescribed dose was $5 \mathrm{~Gy}$ at $20 \mathrm{~mm}$ of the applicator (Figure 5). The

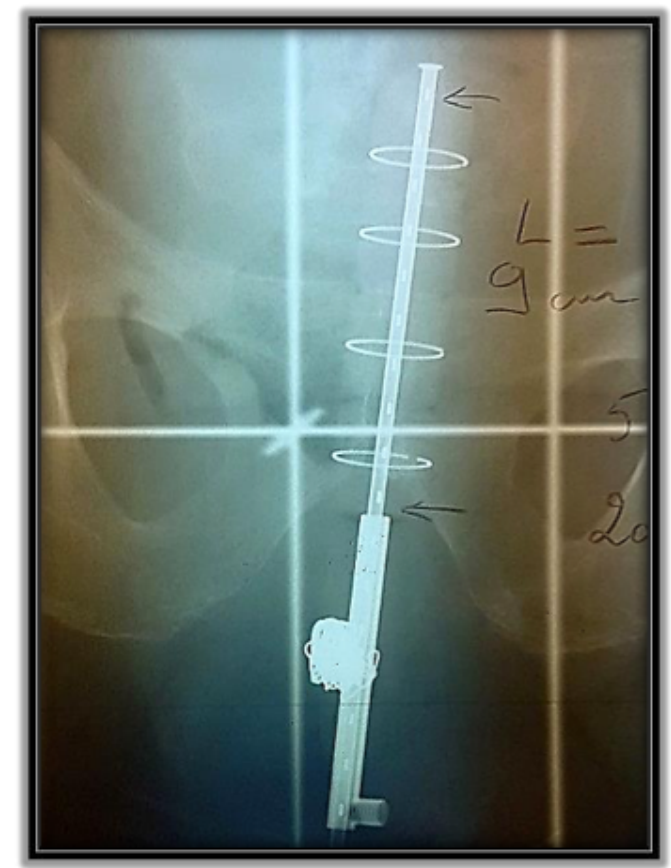

Figure 3. Endorectal applicator. Length $=9 \mathrm{~cm}$; diameter $=2 \mathrm{~cm}$; anal lead cover. 


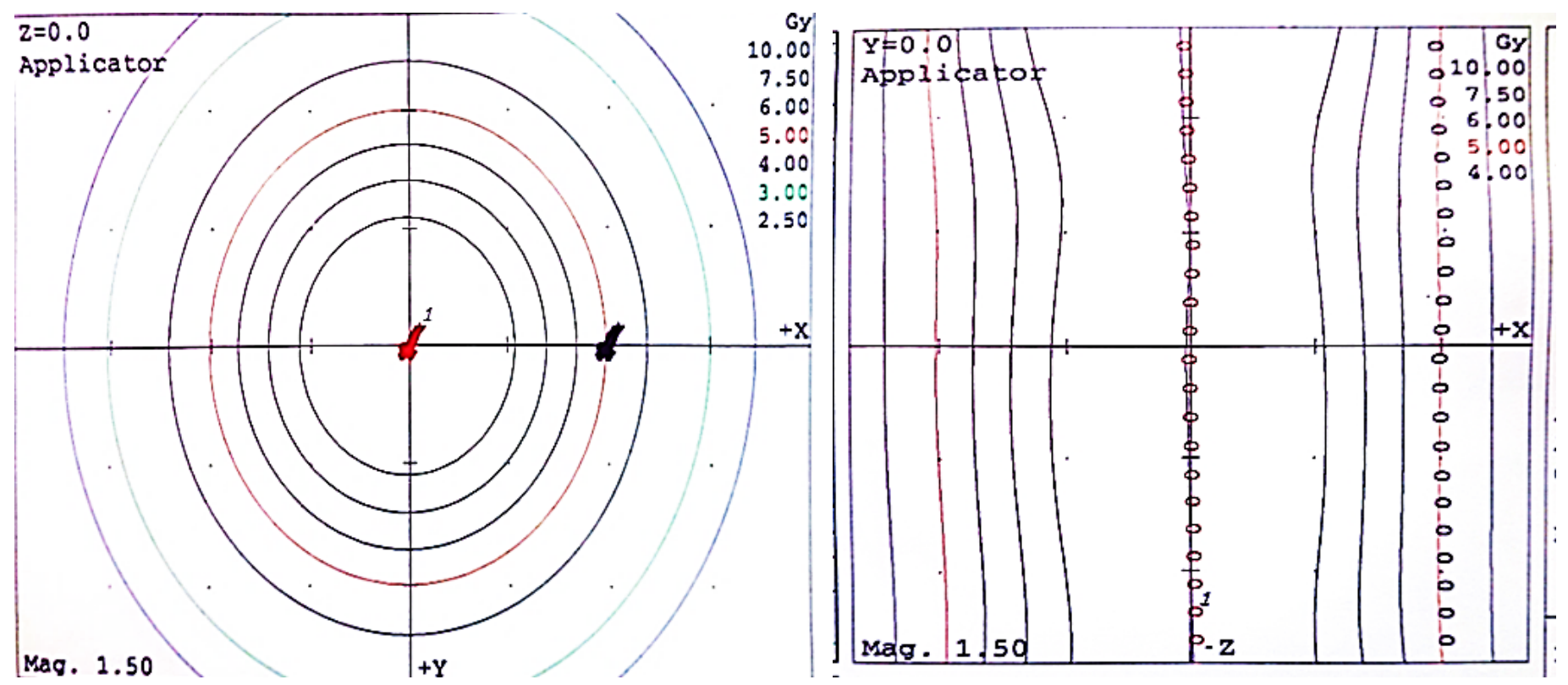

Figure 4. Dosimetry high dose rate brachytherapy. Dose $=5 \mathrm{~Gy}$ at $20 \mathrm{~mm}$; length $=9 \mathrm{~cm}$; position separation=2.5 mm. Dose $=5 \mathrm{~Gy}$ at $20 \mathrm{~mm}$; length=9 $\mathrm{cm}$; position separation=2.5 $\mathrm{mm}$.

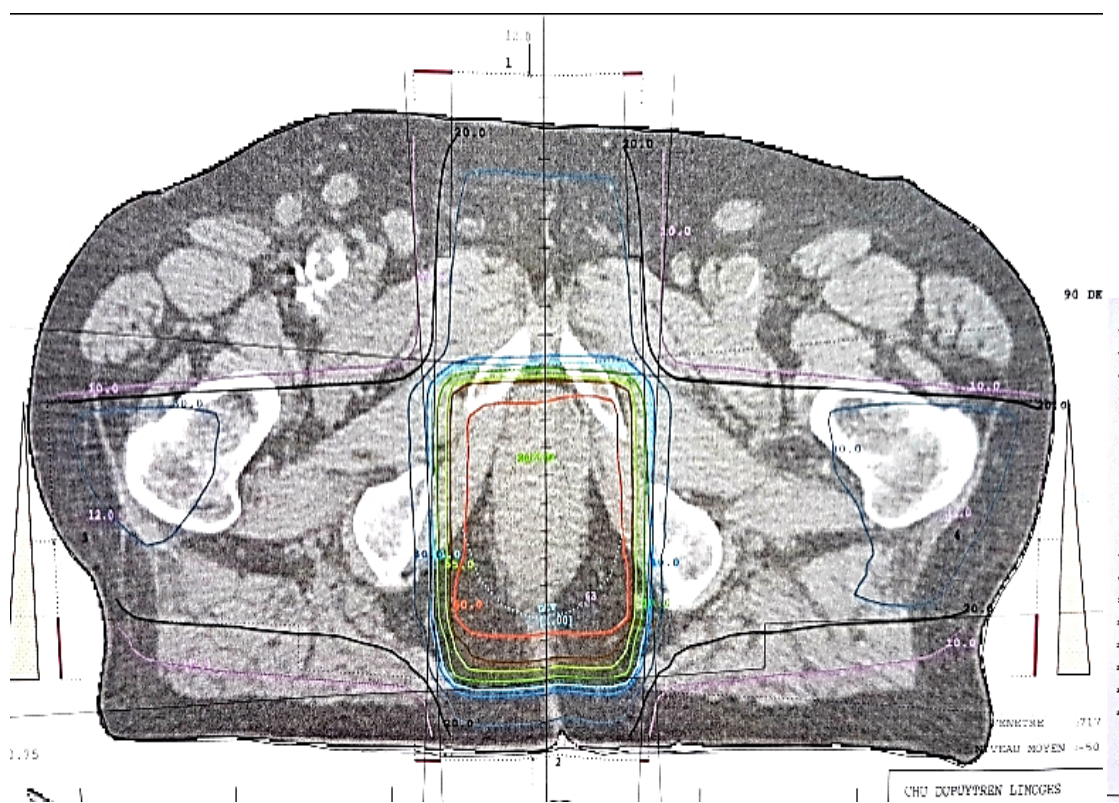

$\begin{array}{lll}\text { Rectum } & 79.14 & \mathrm{~cm} 3 \\ \text { ptv } & 444.10 & \mathrm{~cm} 3 \\ \text { Vessie } & 278.41 & \mathrm{~cm} 3 \\ \text { Prostate } & 76.69 & \mathrm{~cm} 3 \\ \text { tete fed } & 140.67 & \mathrm{~cm} 3 \\ \text { Vesicules } & 9.62 & \mathrm{~cm} 3\end{array}$

Figure 5. Dosimetry, conformal external beam radiotherapy. Dose PTV=60 Gy; anterior, posterior and two lateral beams; deriving dose-volume histogram.

biologically effective dose for the four applications ( $\alpha / \beta$ rectum: 5$)$ was 40 Gy and the equivalent dose (EQD2) was $28.57 \mathrm{~Gy}$.

A 1-month clinical evaluation was used to assess his performance status, rectal bleeding, dose-escalade analgesic and improvement in quality of life.

\section{Results}

The high-dose rate brachytherapy was immediately well tolerated with no acute complications. Moreover, there was no problem of compliance because of the short duration of this treatment, which could be easily performed. The patient described an overall complete response of his symptoms at 1 month. The bleeding had stopped, and he had been withdrawn from opioids. He regained his quality of life with performance status 1 and had maintained independence at home.
Nevertheless, he died of a sepsis 2 months after discharge from our palliative unit. The clinical presentation was necrotizing fasciitis of the perineum for which he had received palliative care and sedation.

\section{Discussion}

External-beam radiotherapy is a common curative and palliative treatment for rectal cancer. It is aimed at reducing the tumour and relieving patients of symptoms such as bleeding, pain and compression. Side effects and treatment modalities are severe for these fragile patients in whom the objective is to improve their quality of life. Moreover, palliative external-beam radiotherapy cannot be performed safely because of the overall radiation dose delivered to the tissues in patients who have previously received external-beam radiotherapy. Brachytherapy is a way to deliver a high dose to the tumour, while 
limiting radiation to surrounding tissues and organs. In addition, high-dose brachytherapy can be performed a few times per week for a couple of weeks, but it can also be just a one-time procedure, which is more comfortable for patients. Some studies have been published concerning endoluminal or interstitial brachytherapy, first with radium (1930-1950) replaced by iridium-192, and most of the results were palliative. Data are predominantly old and analysed from a small number of patients. First, high-dose rate brachytherapy can be safely used after external-beam radiotherapy in selected patients with rectal adenocarcinoma, to provide symptom relief with acceptable toxicity [1-2]. We identified several studies in an internet search. In the Corner study [3] rectal bleeding was controlled in $63 \%$ of advanced inoperable rectal carcinomas; the median duration of the symptom response was 3 months, using a single 10 Gy dose. A retrospective study [5] demonstrated improved bleeding in the palliative group $(n=22)$ with a $64 \%$ response rate obtained, including $57 \%$ complete responses. A mucous discharge was reported in $64 \%$ of cases with $28 \%$ complete responses. The median duration of the response was 7 months. Using a single channel catheter with interchangeable shields as the endoluminal applicator offers better dose homogeneity [6]. A vaginal/rectal cylinder applicator is recommended. Nevertheless, this kind of applicator is not allowed for stenotic tumours, but a small applicator, as used for oesophageal or bronchial brachytherapy, can be used (diameter $=2-4 \mathrm{~mm}$ ). The PTV can only cover the area causing major symptoms. According to Corner's study, palliative treatments are given predominantly with a single $10 \mathrm{~Gy}$ dose. Late toxicities, such as rectal ulcers, strictures and fistulas have been reported, including fewer than one-quarter of the patients, but there are no details about their comorbidities. The aim of using endoluminal brachytherapy is to improve quality of life during and after treatment while limiting side effects. Quality of life was evaluated in an active study that used endoluminal brachytherapy as a preoperative treatment [5] in these patients. Our case died of necrotizing fasciitis of the perineum, which is a rare disease with a high mortality rate. It is an infection with a polymicrobial aetiology and a fast progression that causes advancing necrotic inflammation of tissues. Damage to the deep tissues results in vascular occlusion and ischemia. Perineal necrotizing fasciitis is an emergency that requires a combined surgical/therapeutic management [6]. The disease is facilitated by cardiovascular comorbidities, such as peripheral arterial disease and radiation therapy. Two cases of necrotizing fasciitis of the perineum after radiation have been reported in the literature $[7,8]$. Both were rectal cancer cases with cardiovascular comorbidities and post-radiotherapy complications, such as chronic radiodermatitis (total dose $\geq 50 \mathrm{~Gy}$ ). We deduced that high-dose radiation in patients with severe cardiovascular comorbidities is not recommended.

\section{Conclusion}

Endoluminal high-dose rate brachytherapy is a very effective palliative treatment after external-beam radiotherapy for rectal adenocarcinoma. There are no guidelines for the optimal dose per fraction. A single dose of $10 \mathrm{~Gy}$ is mainly used, with an applicator of adapted dimensions. This technique can be easily performed, and is relatively comfortable for patients, with a short treatment duration. The aim is to reduce bleeding, pain and obstruction, which significantly improves patient quality of life. However, toxicities must be considered, and this case shows that patients should be selected based on their comorbidities and tumour characteristics. Endoluminal high-dose rate brachytherapy may be less effective for high rectal and thick tumours. Precautions must be taken regarding patients with severe peripheral arterial disease previously treated by external-beam radiotherapy, and the overall radiation dose must be considered.

\section{References}

1. Begum N, Asghar AH, N S, Khan SM, Khan A (2003) High dose rate intraluminal brachytherapy in combination with external beam radiotherapy for palliative treatment of cancer rectum. J Coll Physicians Surg Pak 13: 633-636. [Crossref]

2. Chuong MD, Fernandez DC Shridhar R, Hoffe SE, Saini A, et al. (2013) High-dose-rate endorectal brachytherapy for locally advanced rectal cancer in previously irradiated patients. Brachytherapy 12: 457-462. [Crossref]

3. Hoskin PJ, de Canha SM, Bownes P, Bryant L, Glynne Jones R (2004) High-doserate afterloading intraluminal brachytherapy for advanced inoperable rectal carcinoma. Radiother Oncol 73: 195-198. [Crossref]

4. Hansen JW, Jakobsen A (2006) The importance of applicator design for intraluminal brachytherapy of rectal cancer. Med Phys 33: 3220-3224. [Crossref]

5. Woodcock NP, Sebag-Montefiore D, Mannion RA, Alexander DJ, Miller GV (2006) Necrotising Fasciitis Secondary to Locally Advanced Rectal Cancer. Ann R Coll Surg Engl 88: W7-W9. [Crossref]

6. Park SH, Choi JR, Song JY, Kang KK, Yoo WS, et al. (2012) Necrotizing fasciitis of the thigh secondary to radiation colitis in a rectal cancer patient. $J$ Korean Soc Coloproctol 28: 325-329. [Crossref]

7. Kolotas C, Röddiger S, Strassmann G, Martin T, Tselis N, et al. (2003) Palliative interstitial HDR brachytherapy for recurrent rectal cancer. Implantation techniques and results. Strahlenther Onkol. 179: 458-63. [Crossref]

8. Bishop AJ, Gupta S, Cunningham MG, Tao R, Berner PA, et al. (2014) Interstitial Brachytherapy for the Treatment of Locally Recurrent Anorectal Cancer. Ann Surg Oncol 134: 1929-1930. [Crossref]

Copyright: (C2019 Alati A. This is an open-access article distributed under the terms of the Creative Commons Attribution License, which permits unrestricted use, distribution, and reproduction in any medium, provided the original author and source are credited. 